\title{
An Experience with Augmenting a Mirror as a Personal Ambient Display
}

\author{
Kaori Fujinami ${ }^{1}$ and Fahim Kawsar ${ }^{2}$ \\ 1 Department of Computer, Information and Communication Sciences, \\ Tokyo University of Agriculture and Technology, Japan \\ 2 Department of Computer Science, Waseda University, Japan \\ fujinami@cc.tuat.ac.jp, fahim.kawsar@gmail.com
}

\begin{abstract}
In this paper, we describe a case study on augmenting a daily object, mirror, for a personalized ambient display. The mirror displays information relevant to a person in front of it on the periphery of his/her sights. The interaction methods with the mirror were evaluated by a Wizard-of-Oz method and an in-situ experiment. The subjects preferred more controllability over the proactive functionalities and the understandability of information than the passive and the reflective nature of a mirror.
\end{abstract}

\section{Introduction}

Due to the advancement of technologies, our daily lives and environments are full of computational devices and a heavy amount of information. Not only traditional computers, but also small palm sized devices like cellular phones and personal digital assistants (PDA) have network connectivity and offer various information like weather forecasting, today's schedule, etc. This allows people to acquire information anytime anywhere. Also, a new variation of traditional displays like ambient displays[11] are installed into our daily living for showing information to a user. However, if these devices do not provide information in an appropriate way, i.e. considering proper timing, location, identity, and intuitiveness, then computation devices cannot offer comfortable services, because people feel inconvenient to their daily living, and the inconvenience makes them confused how to use the technologies. This means that a user requires to have some efforts to acquire useful information. Offering appropriate information in respective situations is a major research topic in ubiquitous/pervasive computing environments, and this is known as context-awareness.

A mirror has been utilized by people since ancient days[8]. It reflects physical objects appearing in front of it. Often we become inquisitive about our physical appearance by looking at a mirror. Moreover, while using a mirror we can also see and comprehend what is happening in the backgrounds, e.g. someone is passing, a kettle is boiling, etc. This reflective nature is essential for a mirror. We usually stay in front of a mirror for a period of time, which suggests the acceptability of presenting information on the surface of the mirror. In our earlier study[2], 
an augmented mirror was realized by attaching a translucent mirror board on an ordinary computer monitor, where a bright color behind is seen through while an object in front of the board is reflected in the dark colored area. The mirror, AwareMirror, presents information relevant to a person in front of it. We can change our behavior once we notice some unwanted/unknown situation. Our initial design principle is to preserve the metaphor of a daily object intact while adding some values. We have considered that such seamless augmentation reduces the burden of accessing digital world. The primary user feedbacks on the contents and the user identification method were mostly positive. In this paper, we focus on the extensive evaluation of the interaction methods with the mirror. That aims to investigate a user's attitude towards the trade-off between active involvement with augmented functionalities and passive utilization of the traditional reflective nature, which has not been revealed by existing work and products that deal with such an augmented mirror[3][6].

The following sections are organized as follows. Section 2 describes the overview of the AwareMirror system. In section 3, user studies by a Wizard-of-Oz (WoZ) method and an in-situ experiment are presented. Related work is examined in section 4 , and finally section 5 concludes the paper.

\section{AwareMirror System}

In this section, the AwareMirror system is introduced. Then, a discriminative feature of the system, i.e. two modes of information rendering, is reviewed for later evaluation. The evaluation is the contribution of this paper. For more detail on the design rationale and initial evaluation of the system, please refer to [2].

\subsection{Overview}

AwareMirror displays information relevant to a person in front of it on the periphery of his/her sights. A user is implicitly identified in a privacy aware manner. This is an important aspect for augmenting a mirror since the place around a mirror is highly privacy sensitive. We have determined to integrate three types of low level information, rather than utilizing a single and rich data source like a video camera. They are 1) the existence of a person in front of a mirror using an infra-red range finder, 2) the collocation of a personal (unshareable) artefact with a mirror, and 3) utilization of the personal artefact. Context information can be retrieved from a user's daily activities in a natural way. Such everyday artefacts include a safety razor (an electric shaver), a toothbrush, a comb, etc. We have decided to use a toothbrush for detecting a user because it is gender neutral and utilized by almost everyone.

We brush our teeth, make up, etc., that is normally done in situations where we are considering immediate events or something important over the day to come in the morning. We consider information related to immediate event is useful in such a case. We also often change our decision if someone tells us about weather forecasting, traffic accident, etc. Considering these facts, we have 
selected three types of information to be displayed that can affect our behavior and supports decision making: 1) weather forecasting (state and temperature) at the destination where he/she will go, 2) state of a public transportation system that the person is going to use, and 3) information about the next appointment. These types of information can remind us of taking something required, e.g. umbrella and documents, and offers us the opportunity to take an alternative route to go to the destination, or rush ourselves. So, we consider that it is appropriate for information presented on a mirror.

\subsection{Two Modes of Information Rendering}

Although the above listed information is useful, it might disturb a user's primary task if all information appears on the surface of a mirror. Also, privacy sensitive information can be revealed. Therefore, we have decided to apply two types of information rendering and interaction methods with the mirror. We have designed the mirror to start presenting information with abstract images so that a user could perceive the contents at a glance (Fig. 1-(a)). On the other hand, Fig. 1-(b) shows textual messages for clear understanding, where the message describes the image. The numbers in Fig. 1-(a) correspond to the three types of information introduced in the previous section.

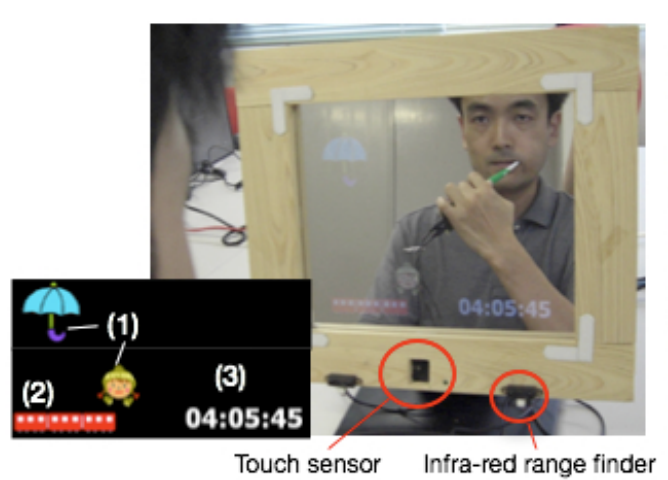

(a) Image-based abstract presentation

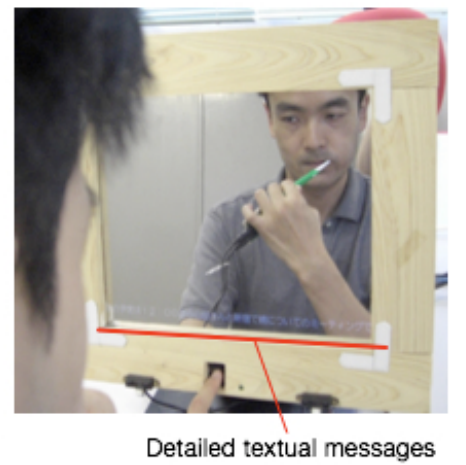

(b) Text-based detailed presentation

Fig. 1. Using AwareMirror: (a) abstract and (b) detailed mode of presentation. Three types of decision supportive information are presented: (1) weather forecasting (state and temperature) at his next destination, (2) state of a public transportation system to be used, and 3) information about the next appointment (time to the event). A user can change the level of abstraction by touching an area on the bottom (b).

There are three presentation modes including the default one. Fig. 2 illustrates the modes, where the solid and dotted lines indicate automatic and manual 
transition, respectively. In the default mode, the mirror does not display anything, so it just looks like a mirror. However, when a person appears in front of AwareMirror and a toothbrush is utilized, then AwareMirror enters into the abstract mode. In the abstract mode, it plays a role of an ambient display, which shows abstract information on the periphery of a user's line of sight. So, in this mode, people use AwareMirror as an ordinary mirror. Abstract information can be represented using images and colors we are familiar with and therefore easy to understand at a glance. This is also a solution that takes into account of the characteristic of the place, in front of a mirror, since some people often take their glasses off while brushing their teeth and washing their faces. Therefore, we consider that it is useful for them to be just notified of some important events.

In the detailed mode, they explicitly use it as a display rather than a mirror. It shows information in more detail using text information. Offering detailed information automatically might break our design philosophy, i.e. keeping the original usage of a mirror intact. It could interrupt a user's current task with sudden appearance of text message. So, we provide the flexibility to change the mode of the usage by him/herself. On entering into the detailed mode, the role of AwareMirror turns into an information display, not an ordinary mirror. This is caused by the person explicitly. Hence, we consider that a feeling of disturbance does not come up in his/her mind. Although the mode is more detailed than the image-based one, we do not intend to complete the information acquisition process of a user within the AwareMirror system. There have already been many media for detailed information like a TV and a website. A user who has more interests in the information presented by the mirror should access them with more efforts. Too much information by the mirror would make the system complex and thus unusable. Role sharing with other media is crucial. We also consider our approach meets the requirements for ambient displays introduced by Mankoff et al[7].: Easy transition to more in-depth information. Both modes automatically return to the default mode on detecting a user's leaving.

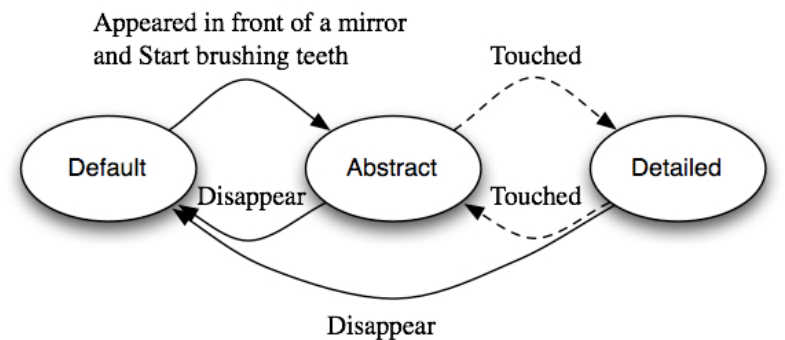

Fig. 2. Transition among default (left), abstract (middle), and detailed mode (right): The solid and dashed lines indicate automatic and manual transition, respectively. 


\subsection{Prototype Implementation}

Fig. 3 illustrates the relationship among the functional components (rectangle) and their implementation (cloud form). The system consists of a sensing system, AwareMirror Controller, personalized contents acquisition, contents rendering, and the presentation mode controlling components. The core functionality is AwareMirror Controller that accepts events from three sources to identify a person who is in front of the target mirror. The existence of a possible user is detected by the change of the distance to a mirror. To identify the person, the detection of a used toothbrush around the mirror is utilized. An RFID tag of an active type is attached to each toothbrush, where the owner ID is identical to the tag ID. A tag reader that is placed near the mirror is responsible for the detection of collocation of the mirror and a toothbrush. A toothbrush is also augmented with an accelerometer to detect the state-of-use, i.e. start brushing or not. As described in section 2.1, three types of information is utilized, that is based on a user's schedule. We have mashed up existing contents to provide real experiences in in-situ experiments. The Contents Rendering component draws the two modes as well as the default one based on the transition shown in Fig. 2. In the experiments, different policies for the contents rendering and presentation mode controlling were implemented for each comparative functionality.

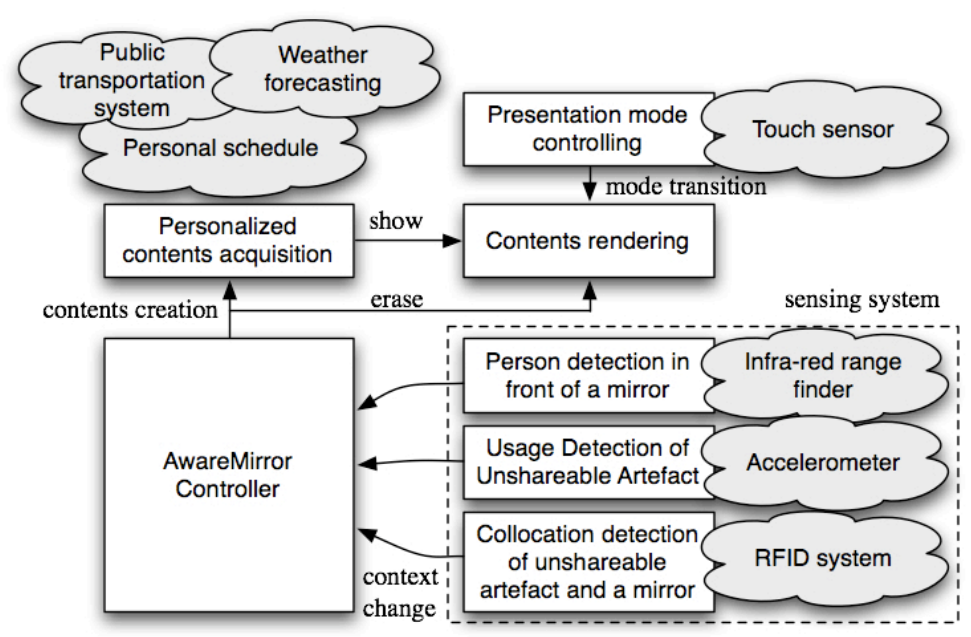

Fig. 3. Functional components and implementation of AwareMirror system

The explicit transition to the detailed mode is realized by a touch sensor embedded into the bottom frame (see Fig. 1-(a)). In our initial prototype, a "zipper" metaphor was utilized, where a variable resistance-based slider was attached to provide a user with tangible interaction to get information, i.e. opening 
for getting detailed information. However, it was not supported by the subjects because of 1) muliple roles and 2) statefulness. A zipper is utilized not only to extract something from inside (digital value), but also to fasten something at a proper position like a zip-up jacket (analog value). This made the subjects confused. Instead, they liked to utilize a touch sensor. Although the duality might be overcome by learning, the statefulness is problematic. AwareMirror turns to the black screen (default mode) when it detects a user's absence in the both abstract and detailed modes. Here, we assume that the zipper remains opened. When a new user comes in front of AwareMirror, it displays abstract information. So, he/she needs to close the zipper first, and then opens the zipper to change into the detailed mode, which makes them confused. Considering these two issues, we have changed to a touch-based interaction. People have already had a mental model for a (stateless) touch button since it is often utilized in controlling electronic appliances, e.g. a TV remote and a PC.

\section{Evaluation}

In this section, the interaction method with the mirror is evaluated with a WoZ method[1] and in-situ experiments.

\subsection{Methodology}

Contrastive functionalities were implemented, and tested together with the original ones described in section 2. The points of the comparison are as follows.

1. Automatic vs. Manual activation of the first (abstract) presentation

2. Automatic vs. Manual transition between abstract and detailed presentation

3. Existence vs. Little-existence of the reflective nature in detailed presentation

4. One phase (only textual message) vs. Two phase information provision

Note that the manual activation of the first scene was realized by touching the same area as the mode transition. Also, in automatic transition case, the second scene appeared after 10 seconds. The version with little reflective nature can be seen in Fig. 4. The message is drawn in black on white background, that reduces the reflection on the surface due to the characteristic of a magic mirror. Therefore, it looks like a traditional computer screen, rather than a mirror.

A WoZ method was applied to obtain comprehensive view while an in-situ experiment was conducted for intimate findings. Fourteen subjects (12 students from outside the authors' laboratories and 2 company employees) were recruited for the WoZ experiment. Each subject was directed to use the system, e.g. start brushing teeth, pressing the touch sensor. An observer actually controlled the system. Then, he/she had an interview session based on a filled survey form.

The in-situ experiments were conducted with totally four persons, i.e. one family (parents and an adult daughter) and a company employee living alone. A working prototype was installed in the living room at each home for 17 and 22 days, respectively. The subjects were asked to change the properties for the 
different experimental conditions by themselves through a simple GUI and a manual. They were also told to change the comparative functionalities just after the previous utilization. We consider the subjects should focus on performing the primary task or utilizing the functionality that is subject to evaluate. So, preparing for the experiment needs to be completed beforehand. Finally, they were interviewed based on a survey form and sheets of paper on which they had written any comments throughout the experiment. In the following discussion, the results of the above two experiments are combined.

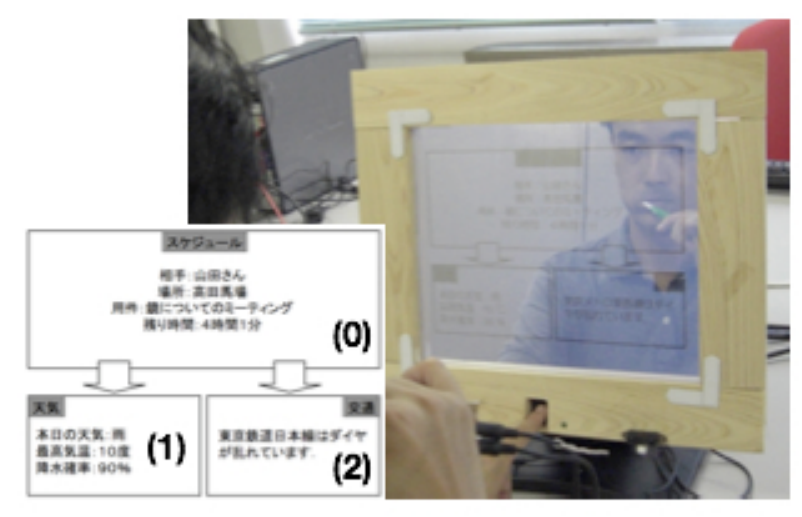

Fig. 4. The contrastive rendering of the detailed presentation, where static textual messages are written in black on white background. The number (1) and (2) correspond to the ones in Fig. 1-(a), while (0) indicates the details of the next appointment rather than the down counter (3) in the previous figure.

\subsection{Results and Discussions}

Activation of Abstract Information Mode: The half of the subjects (7 people) preferred the automatic activation that does not require any explicit direction. The comments are that even simple touching was messy in a busy morning, and sometimes forgettable. This indicates that they preferred to confront the system as an ordinary mirror. Notably, all the four subjects from the in-situ experiments liked this version.

On the other hand, the subjects who liked manual activation needed the rendered information only when they wanted to see it. This comes from their situations where they usually had no specific plan that requires decision making provided by AwareMirror. In that case, the presented information became obtrusive even if it is image-based abstract information. We consider that filtering out only relevant situation for a person by his/her preference or the system's intelligence is needed. For example, an accidental situation of a train to take can be shown while the normal case does not render any information. These facts suggest that preserving a mirror's original functionality is important for all the subjects. 
The other interesting reason is that the unreliable behavior of the system due to a sensing subsystem made them refuse proactive information provision. A subject complained that he had been surprised at sudden activation of the system at midnight, i.e. the abstract mode was presented, since he had been looking his face into the mirror at that time. People may feel monitored.

Transition to Detailed Information Mode: Manual transition was supported by $68.8 \%$ of the subjects ( 11 persons). The major reason for the preference is the feeling of controlling a system. They could not wait for the automatic transition (in 10 seconds) when they had something to know the detail. Also, they did not always wish to see the detailed information. Such user experiences are what we have designed. The subjects who liked automatic transition gave their negative impression on the manual one, i.e. touching with a wet hand and bothering with changing the mode. We consider this is not an affirmative preference in the automatic way. This indicates the request for a new (manual) controlling method that takes into account of the constraint in the lavatory.

Detailed Information Rendering: The full screen version (Fig. 4) was preferred by $68.8 \%$ of the subjects due to readability of presented information. It is realized by static textual messages in black color on the white background. It shows all the information in a screen at a glance, although it has little reflective nature. In contrast, the scrolling text version (Fig. 1) requires a user to follow the message to the end, and thus he/she needs to pay more attention. The reflective nature of the mirror would rather obstacle. The subjects pointed that they noticed the mirror was not functioning during the full screen. But they did not mind it since the time to check the detailed information was short because of the readability. This suggests that the full screen version contributes to reduce the interruption into the primary task consequently. On the contrary, a subject who had negative impression on the full screen version requested to see her face in the whole period of brushing teeth. This indicates the desire to keep the original function intact at any time, that is supportive of our design principle.

Two Phase Information Presentation: Ten subjects (66.7\%) wanted the two phase information presentation, i.e. the detailed information with textual message is presented after a graphical abstract information. The major reasons are that 1) they do not always need detailed information and 2) it is difficult to read the textual message from the beginning in case that they do not wear glasses. The subjects who participated to the in-situ experiment turned to the textual expression mode when they actually wanted to know the detail. Two of the subjects changed their behavior twice based on the information (one for low temperature and one for an accident of public transportation). Such a decision making support during ordinal task is what the mirror is aiming at. Four of the ten subjects actually thought that only the first scene was needed. We consider it is very difficult to render detailed information like the time, location, and estimated recovering time of a train accident. So, finally, we have classified them into 
the group who preferred two phase information presentation as potential members. Furthermore, a subject looked forward to seeing a change in the image. We could say this is interesting and important aspect in a daily life computing paradigm. Such an aspect is basically ignored by a traditional "efficiency-based" computing paradigm. Also in this point, the two phase presentation proved useful.

\section{Related Work}

Regarding the application and the interaction method with an augmented mirror, information that supports decision making like ours has also proposed in [6], where gesture and touching is utilized for the manipulation of presented information. Also, Miragraphy [3] provides more active involvement. Information about clothes and their coordination is shown by the detection of an RFID tag, and the history of daily clothes captured by a video camera is presented. To the best of our knowledge, a user explicitly utilizes the functionalities provided by these systems, that could make a gap between a user and a system. Our interaction method described in section 2.2 would provide very small gap since the involvement is designed to be the extension of the activities in front of an ordinal mirror.

In terms of information representation, an ambient display[11] in the context of tangible user interfaces[5] offers an information using human's peripheral awareness capabilities. Our AwareMirror is also an ambient display in a sense because it allows a person to focus on his/her main tasks while offering information in his/her line of sights. The work of Rodenstein [9] can also be categorized into an ambient display. It applies the opposite notion of a mirror and the biggest characteristic of a window, transparency, to overlap information in cyber space, i.e. short-term weather forecast, onto a view of outside. Information visualization with artistic impression like Informative Art [4] and InfoCanvas [10] suggests an "augmented paintings", which can naturally fit into daily living. Their roles are presenting information from the beginning, while AwareMirror has more strict constraints that it should not change its original usage as a mirror while offering additional values, personalized information.

\section{Concluding Remarks}

A mirror was augmented to present information relevant to a person in front of it. Our initial design principle was to keep the original metaphor of a mirror intact, where 1) presentation starts with an abstract image on identifying a user, 2) detailed information appears/disappears at his/her request, and 3) even the detailed information is rendered in a form that keeps a mirror's reflective feature.

The design principle was evaluated by comparative functionalities. That aimed at investigating a user's attitude towards the trade-off between a) active involvement with the augmentation and b) passive utilization as a traditional mirror. The result shows that the majority of the subjects preferred the two 
phase information provision, the automatic activation and the manual transition. However, realizing the second phase with a mirror's reflective feature was not the majority. These findings suggest that a user likes to confront AwareMirror as an ordinary mirror at the beginning, but once he/she has interests in the information, it should be useful as an information display. Here, a designer of an augmented artefact needs to take care of the controllability of a system and the efficiency of understanding information, rather than adhering to keeping an artefact's original functionality intact. This may sound trivial in a context-aware system design. However, we believe the findings would contribute to update the design principle of a new type of a display that provides information through its original usage.

\section{References}

1. N. Bernsen, H. Dybkjaer, and L. Dybkjaer. Designing Interactive Speech Systems - From First Ideas to User Testing. Springer Verlag, 1998.

2. K. Fujinami, F. Kawsar, and T. Nakajima. AwareMirror: A Personalized Display using a Mirror. In Proceedings of International Conference on Pervasive Computing, Pervasive2005, LNCS 3468, pages 315-332, May 2005.

3. HITACH, Human Interaction Laboratory. Miragraphy. URL: <http://hhil. hitachi.co.jp/products/miragraphy.html> (in Japanese).

4. L. E. Holmquist and T. Skog. Informative art: Information visualization in everyday environments. In GRAPHITE '03: Proceedings of the 1st international conference on Computer graphics and interactive techniques in Australasia and South East Asia, pages 229-235, 2003.

5. H. Ishii and B. Ullmer. Tangible Bits: Towards Seamless Interfaces between People, Bits and Atoms. In Proceedings of Conference on Human Factors in Computing systems, pages 234-241, 1997.

6. T. Lashina. Intelligent bathroom. In Ambient Intelligence technologies for wellbeing at home, a workshop on European Symposium on Ambient Intelligence (EUSAI2004), 2004.

7. J. Mankoff, A. K.Dey, G. Hsieh, J. Kientz, S. Lederer, and M. Ames. Heuristic evaluation of ambient displays. In Proceedings of the conference on Human factors in computing systems, pages 169-176, 2003.

8. Richard L. Gregory. Mirrors in Mind. W. H. Freeman and Company, 1997.

9. R. Rodenstein. Employing the Periphery: The Window as Interface. In Extended abstract of International Conference on Human Factors in Computing Systems(CHI'99), pages 204-205, May 1999.

10. J. Stasko, T. Miller, Z. Pousman, C. Plaue, and O. Ullah. Personalized Peripheral Information Awareness through Informative Art. In Proceedings of 6th International Conference on Ubiquitous Computing(Ubicomp2004), pages 18-35, October 2004.

11. C. Wisneski, H. Ishii, A. Dahley, M. Gorbet, S. Brave, B. Ullmer, and P. Yarin. Ambient Displays: Turning Architectural Space into an Interface between People and Digital Information. In Proceedings of the First International Workshop on Cooperative Buildings (CoBuild'98), pages 22-32, February 1998. 